\title{
Aromatase et infertilité masculine
}

\author{
Serge CARREAU1, Claire de VIENNE2, Lamia SAID1,3, Ali SAAD3, \\ Isabelle GALERAUD-DENIS1,2
}

1 Université de Caen, EA 2608, USC INRA, F-14000 Caen, France

2 CHRU, Biologie de la Reproduction, F-14000 Caen, France

3 Service de Cytogénétique et Biologie de la Reproduction,

Hôpital F. Hached, Sousse, Tunisie

\section{RESUME}

Chez la plupart des mammifères étudiés jusqu'à ce jour, l'aromatase est présente dans les cellules somatiques et les cellules germinales du testicule. Ainsi chez le rat adulte nous avons démontré l'existence d'une aromatase fonctionnelle, notamment dans les spermatocytes pachytènes et les spermatides rondes, qui assure une production d'estrogènes équivalente à celle des cellules de Leydig.

Chez l'homme, outre les cellules de Leydig nous avons mis en évidence l'aromatase dans les cellules sexuelles immatures et dans les spermatozoïdes éjaculés. Plus précisément, l'expression de l'aromatase diminue de $30 \%$ dans la fraction de spermatozoïdes immobiles comparée à celle contenant les spermatozoïdes mobiles d'un même prélèvement. Sur des échantillons issus de patients asthénospermiques, tératospermiques, et asthénotératospermiques, les taux de transcrits de l'aromatase sont respectivement diminués de 44,52 , et $67 \%$ par rapport à celui des patients normospermiques. De plus, ces taux de transcrits sont fortement corrélés $(r=-0,64)$ aux pourcentage d'anomalies morphologiques du spermatozoïde et notamment à celles touchant la tête et l'acrosome en particulier.

Des techniques immunocytochimiques associées à la microscopie confocale indiquent une forte localisation de l'aromatase au niveau du flagelle et de la pièce intermédiaire. Les altérations de la fertilité chez l'homme déficient en aromatase et nos résultats suggèrent une participation probable des estrogènes dans la mobilité et la survie des spermatozoïdes. De plus, la corrélation du taux de transcrit au degré de tératospermie ainsi qu'une localisation partielle de l'aromatase au niveau de la tête du spermatozoïde semblent confirmer l'implication de l'aromatase au cours de la réaction acrosomique.

Il est donc envisageable d'utiliser l'aromatase comme marqueur du bon déroulement de la spermatogenèse et comme paramètre pour estimer la fécondance. En somme, dans le contrôle des fonctions de reproduction chez le mâle, outre les gonadotrophines et la testostérone, les estrogènes ont un rôle certainement plus complexe que cela avait été suggéré, compte tenu de l'expression particulière de l'aromatase et de la présence de récepteurs aux estrogènes dans les cellules testiculaires et les spermatozoïdes en particulier. L'infertilité est un problème de société qui concerne tous les pays et donc les études menées sur les empreintes nucléaires doivent permettre de mieux comprendre certains aspects de la physiopathologie de la reproduction masculine.

Mots clés : aromatase, estrogènes, spermatozoïde, testicule, homme, fertilité

Correspondance :

Dr Serge CARREAU - Université de Caen, EA 2608-

USC2006 INRA, IFR 146, Esplanade de la Paix-14032 Caen

- Tel 0231565488 - Fax 0231565120 -

Email serge.carreau@unicaen.fr 


\section{INTRODUCTION}

L'aromatase est l'unique enzyme catalysant de manière irréversible la transformation des androgènes en estrogènes. Chez l'homme cette enzyme est localisée dans le réticulum endoplasmique, elle est codée par un gène unique sur le chromosome 15 (Cyp19) qui contient 18 exons dont 9 sont codants. Outre ces exons, 9 exons supplémentaires noncodants dérivés de l'exon I régulés par des facteurs spécifiques permettent un contrôle efficace de l'expression de l'aromatase ; cependant une seule protéine de $55 \mathrm{kDa}$ est décrite chez l'homme [34, 35]. Elle est impliquée dans le développement, la différenciation sexuelle et le comportement, la reproduction, les métabolismes osseux et lipidique, le fonctionnement du cerveau mais aussi dans de nombreux développements tumoraux ; son importance est donc capitale chez l'homme.

Le testicule des mammifères assure deux fonctions essentielles : la synthèse des hormones stéroïdes et la production des gamètes mâles. Le contrôle des fonctions testiculaires est assuré principalement par les hormones gonadotropes et la testostérone auxquelles s'ajoutent de nombreux facteurs produits localement et parmi eux les estrogènes (revues de Carreau et al. $[10,11]$ et de O'Donnell et al. [27]). De très nombreux travaux sont venus confirmer la synthèse d'estrogènes testiculaires chez l'homme (revues de Lambard et al. [23, 24]); de plus il est important de rappeler que dans la veine spermatique le taux d'estrogènes est environ 50 fois plus élevé que dans le sang périphérique [19].

Outre leur rôle de rétrocontrôle central sur l'axe hypothalamohypophysaire, les estrogènes jouent un rôle éminent in situ et de fait il apparaît que les estrogènes sont tout aussi important chez l'homme que chez la femme compte tenu notamment des données acquises ces dernières années chez les patients déficients en aromatase (revue de Rochira et al. [32]). Un autre point à souligner concerne le traitement des femmes au diéthylstilbestrol et son impact délétère sur le tractus génital (cryptorchidie, hypospadias) du fétus mâle ; s'ajoutent à cela les observations multiples concernant l'incidence des perturbateurs endocriniens et particulièrement les xéno-estrogènes chez l'homme (revue de Toppari et al. [37]).

Toutes ces données s'expliquent en particulier grâce aux récentes découvertes mettant en évidence une production locale d'estrogènes et surtout par une large présence des récepteurs aux estrogènes tout au long du tractus génital mâle (revues de Carreau et al. [13, 14]).

\section{L'AROMATASE DANS LES CELLULES TESTICULAIRES}

\section{Mise en évidence d'une source d'estrogènes dans les cellules germinales des rongeurs}

En fait, jusque dans les années 90 la distribution cellulaire de l'aromatase dans les cellules testiculaires des mammifères a suscité de nombreuses controverses. II est connu depuis longtemps que l'aromatase est localisée dans les cellules de Leydig [10]. Cependant chez les rongeurs de très nombreux travaux ont démontrés une expression cellulaire variable en fonction de l'âge avec une prédominance sertolienne chez le rat immature puis une expression leydigienne chez l'adulte.

Pour notre part, nous avons chez le rat adulte démontré que les cellules germinales produisent de l'estradiol, et plus précisément nous avons observé que les taux d'ARNm spécifiques de l'aromatase étaient plus élevés dans les spermatocytes pachytènes que dans les spermatides rondes (Figure 1). Al'inverse, l'activité enzymatique (transformation

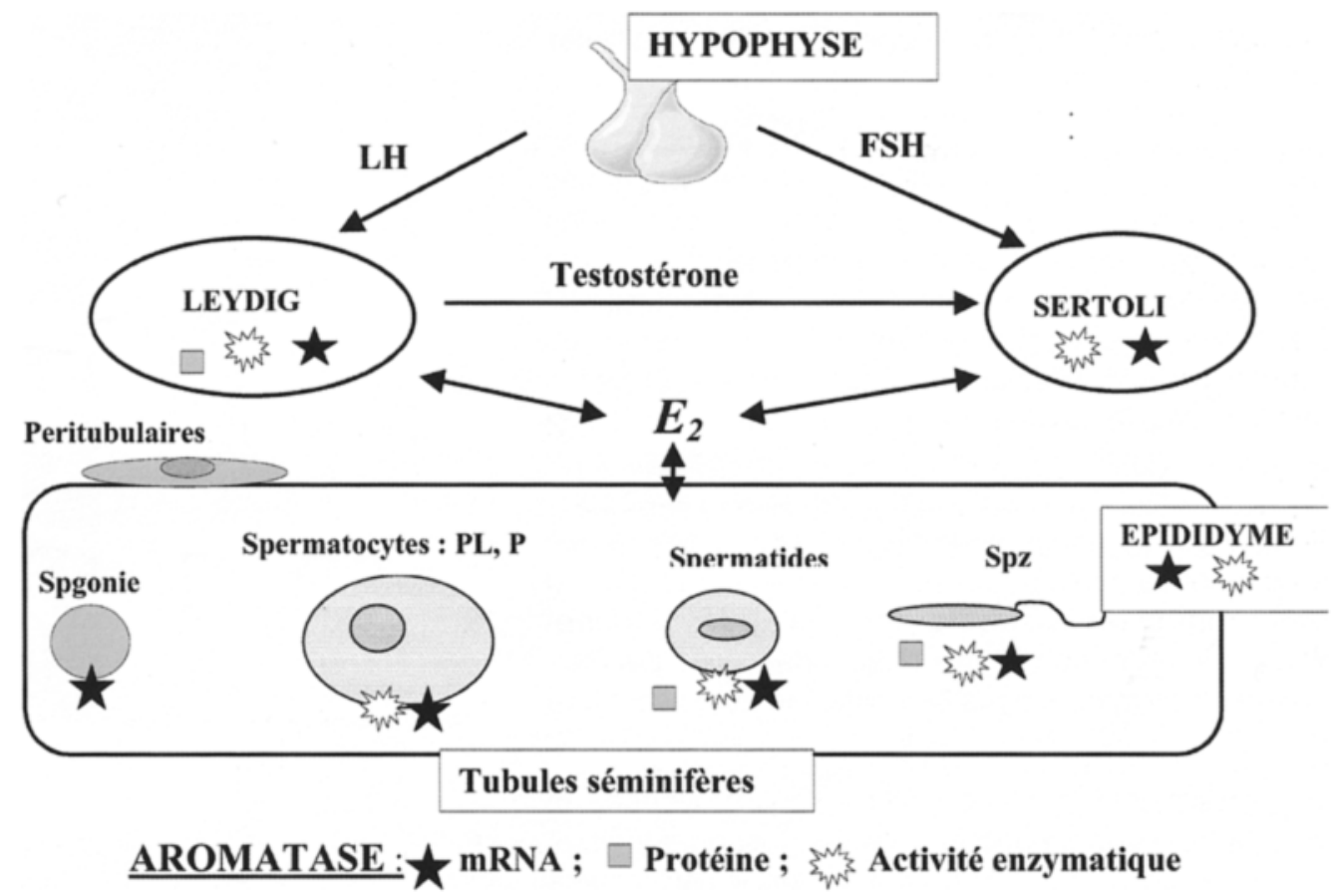

Figure 1 : Source des estrogènes dans le testicule du rat adulte. 
d'androgène en estrogène) est plus intense dans les cellules haploïdes, y compris les spermatozoïdes testiculaires, que dans les cellules plus jeunes ; cette observation a été confirmée par immunohistochimie où une plus forte immunoréactivité vis à vis de l'aromatase a été enregistrée dans les spermatides allongées [25].

La cellule de Sertoli adulte exprime l'aromatase mais les cellules germinales répriment la synthèse d'estrogènes ce qui au total amène à ce constat : l'activité aromatase testiculaire réside pour au moins $50 \%$ dans les cellules germinales (revue de Carreau et al. [12]). Au total nous avons pu démontrer que les cellules testiculaires somatiques (excepté les cellules péritubulaires) mais aussi les cellules sexuelles ont la capacité de synthétiser des estrogènes [12].

\section{L'aromatase dans les cellules sexuelles de l'homme}

Chez l'homme au niveau testiculaire, l'aromatase a été décrite dans les cellules de Leydig $[7,29]$; de plus in vitro, une activité aromatase a été démontrée non seulement dans les cellules de Leydig purifiées mais aussi dans des cultures primaires de cellules de Sertoli (revue de Carreau [9]).

A partir de prélèvements de plasma séminaux d'hommes fertiles, nous avons tout d'abord recherché la présence de l'aromatase (ARNm et protéine biologiquement active) dans les spermatozoïdes. Dans un second temps, en ayant comme hypothèse que l'aromatase pourrait servir de marqueur de la qualité du spermatozoïde (en particulier la mobilité), nous avons mesuré la quantité d'ARN messagers du P450arom dans les fractions enrichies en spermatozoïdes mobiles et dans celle des spermatozoïdes immobiles obtenues à partir du même éjaculat. Puis compte tenu qu'aucune information n'était disponible sur la présence d'aromatase dans les cellules germinales humaines immatures, des préparations hétérogènes de spermatocytes et de spermatides ont été obtenues à partir d'échantillons présentant un nombre élevé de cellules rondes [22].

Nous avons mis en évidence la présence d'ARNm codant pour l'aromatase dans tous les échantillons de sperme purifiés sur gradient discontinu de Puresperm 8 [21]. Nous avons également observé une diminution significative $(30 \%) \mathrm{du}$ rapport des taux de transcrits aromatase/GAPDH (glycéraldéhyde-3-phosphodéshydrogénase) dans les spermatozoïdes immobiles comparé aux mobiles. Dans les cellules germinales immatures, nous avons détecté les ARNm de l'aromatase par RT-PCR et visualisé l'aromatase à 49 $\mathrm{kDa}$ en Western-blot. Nos résultats sont en accord avec ceux d'Aquila et al. [2] qui ont démontré la présence d'aromatase dans un " pool de plasma séminaux » et confirment aussi les observations en immunohistochimie faites par Turner et al. [38] sur la présence d'aromatase dans les cellules germinales et notamment dans la gouttelette cytoplasmique [33].

Puis, très récemment, à partir d'échantillons provenant de patients infertiles (asthénospermiques, tératospermiques, et asthénotératospermiques), nous avons quantifié l'aromatase (et la GAPDH) par PCR en temps réel, et les résultats montrent une chute importante des transcrits de l'aromatase $(44,52$ et $67 \%$ ) respectivement par rapport à celui des patients normospermiques. De plus les anomalies morphologiques les mieux corrélées à cette baisse affectent plus la tête (et en particulier l'acrosome : $r=0,56 ; p<0,001$ ), que le flagelle $(r=0,42 ; p<0,01)$ ce qui suggère une implication de l'aromatase dans la qualité de ce gamète mâle (Said, Galeraud-Denis, Carreau, en préparation).

Avec une approche en microscopie confocale, nous avons ensuite précisé la localisation de l'aromatase dans le spermatozoïde de patients normospermiques et tératospermiques (anomalies fréquentes de l'acrosome) en utilisant différents marqueurs du spermatozoïde (DAPI pour le noyau, CD46 pour l'acrosome, tubuline pour le flagelle et le Mitotracker pour les mitochondries).

Concernant les patients normospermiques (Figure 2), l'aromatase est localisée principalement dans la pièce intermédiaire et la région équatoriale de la tête, une localisation de moindre intensité est observée dans le flagelle ce qui est en accord avec les résultats d'Aquila et al. [2] obtenus en Western blot. Chez les tératospermiques, l'aromatase au niveau de la pièce intermédiaire est corrélée à la morphologie ce qui est en accord avec les pourcentages d'anomalies versus taux de transcrits du P450arom $(r=-0,64)$. Par ailleurs nos résultats préliminaires (Galeraud-Denis, de Vienne, Carreau, European Congress of Andrology, Rome 2008) mettent en évidence au niveau équatorial une proportion de spermatozoïdes marqués à l'aromatase 2 fois plus faible que dans les spermatozoïdes des patients normospermiques $(44 \%)$.

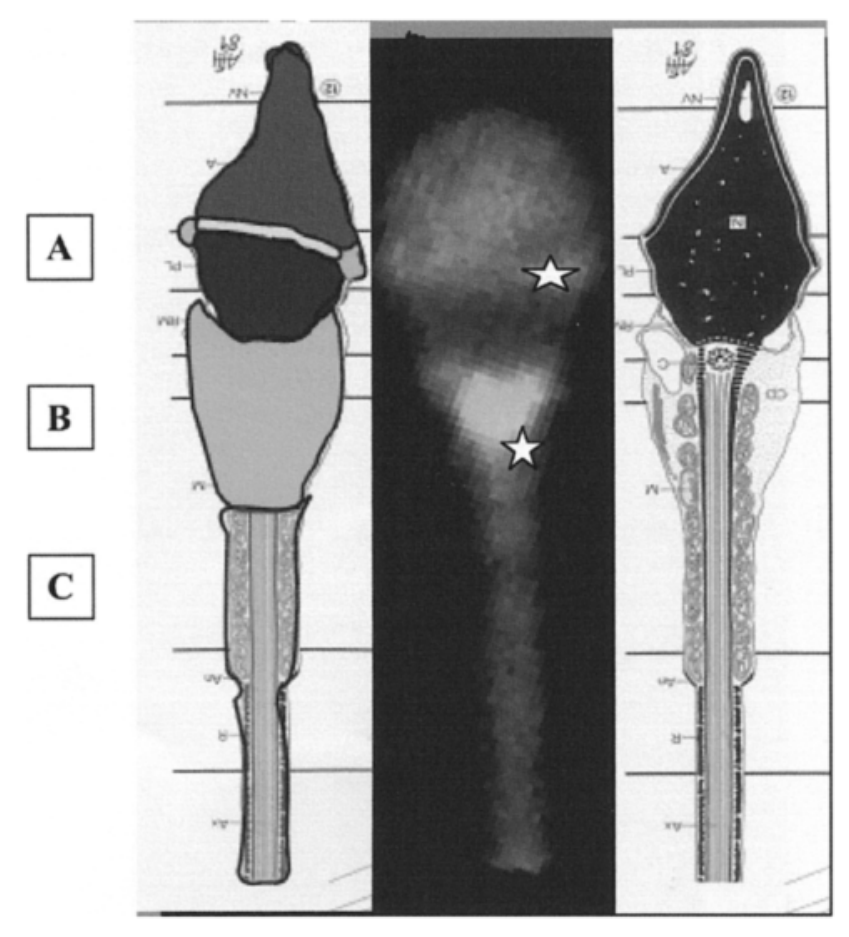

Figure 2 : Localisation de l'aromatase dans le spermatozoïde humain normal. Ultrastructure du spermatozö̈de d'après Holstein \& Roosen-Runge (1981). Observations en microscopie confocale des différentes régions du spermatozoïde : Aromatase : A) Région équatoriale) , B) pièce intermédiaire, C) flagelle. 
En outre, l'aromatase a été immunolocalisée dans les cellules épithéliales au niveau des canaux efférents et de la tête proximale de l'épididyme humain [8]. Des résultats identiques ont été rapportés chez le singe Rhésus avec la présence de 2 transcrits de l'aromatase (dont l'un tronqué) dans le testicule et l'épididyme. De plus l'activité enzymatique est identique dans les 2 tissus mais plus élevée dans la tête de l'épididyme par rapport aux autres régions de cet organe [31].

\section{DISCUSSION et PERSPECTIVES}

Les modèles expérimentaux ou les déficits génétiques en estrogènes (ArKO et ERKO) sont venus conforter les recherches sur l'aromatase et le rôle des estrogènes chez l'animal bien sûr mais également chez l'homme [13, 27, 32]. Dans l'Endocrinologie de la Reproduction chez le Mâle, outre les gonadotrophines et la testostérone, les estrogènes agissent également à différents stades de la spermatogenèse ainsi que dans la spermiogenèse. Leur rôle est certainement plus complexe que cela avait été suggéré, compte tenu de l'expression particulière de l'aromatase et de la présence de récepteurs aux estrogènes dans les cellules testiculaires et les spermatozoïdes en particulier $[4,16,26,36]$.

II faut rappeler qu'en 1995, Gunasegaram et al. [18] avaient décrit l'existence d'une production d'estradiol à partir de prégnénolone dans les spermatozoïdes humains. Compte tenu de la présence de récepteurs aux androgènes [5], la réalité fonctionnelle d'une aromatase biologiquement active dans les spermatozoïdes est évidente. De plus cette localisation de l'aromatase dans la pièce intermédiaire et le flagelle du spermatozoïde humain corrobore les études menées sur sa fonctionnalité via la synthèse d'estrogènes. Ces derniers activent des voies non génomiques (AMPc/PKA,
ERK1/2, PI3kinase) via leur liaison à des récepteurs membranaires [26]. La voie AMPc/PKA est impliquée dans la mobilité du spermatozoïde via la phosphorylation des effecteurs (dynéine et AKAP) présents dans la pièce intermédiaire et le flagelle [4].

Les altérations de la fertilité chez l'homme déficient en aromatase [32] et nos résultats montrant une diminution de l'expression de cet enzyme dans les spermatozoïdes immobiles, et cette diminution est encore plus nette patient chez les patients asthénotératospermiques, ceci suggèrerait donc la participation des estrogènes (et des gènes sous leur contrôle) dans la mobilité des spermatozoïdes comme cela avait été rapporté il y a plus de 20 ans $[6,20]$. Par ailleurs le rôle anti-apoptotique in vitro des estrogènes est démontré sur les cellules germinales humaines [30].

II est donc envisageable d'utiliser l'aromatase comme marqueur du bon déroulement de la spermatogenèse (Figure 3) ; de plus, les estrogènes étant concernés dans la survie et la mobilité du spermatozoïde [30], l'aromatase pourrait servir de paramètre pour estimer la fécondance et peut-être aussi dans les premières étapes du développement embryonnaire [28]. Ainsi plusieurs étapes de la maturation des cellules germinales sont sans doute contrôlées par les estrogènes produits localement (revues de Akingbemi [1] et Carreau et al. $[12,14]$ ) mais il reste à préciser les mécanismes d'action de ces hormones femelles (voies membranaire et/ou génomique ; $[4,26])$ et peut-être mitochondriale où des récepteurs aux estrogènes, notamment de type bêta, sont présents et interviennent non seulement dans les processus énergétiques mais aussi dans de nombreuses fonctions cellulaires (revue de Chen et al. [15]).

\section{Testostérone}
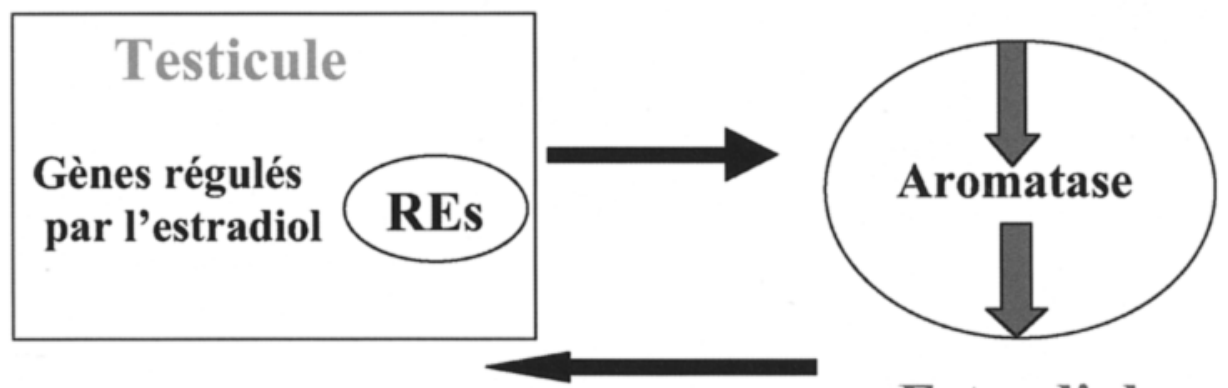

Estradiol

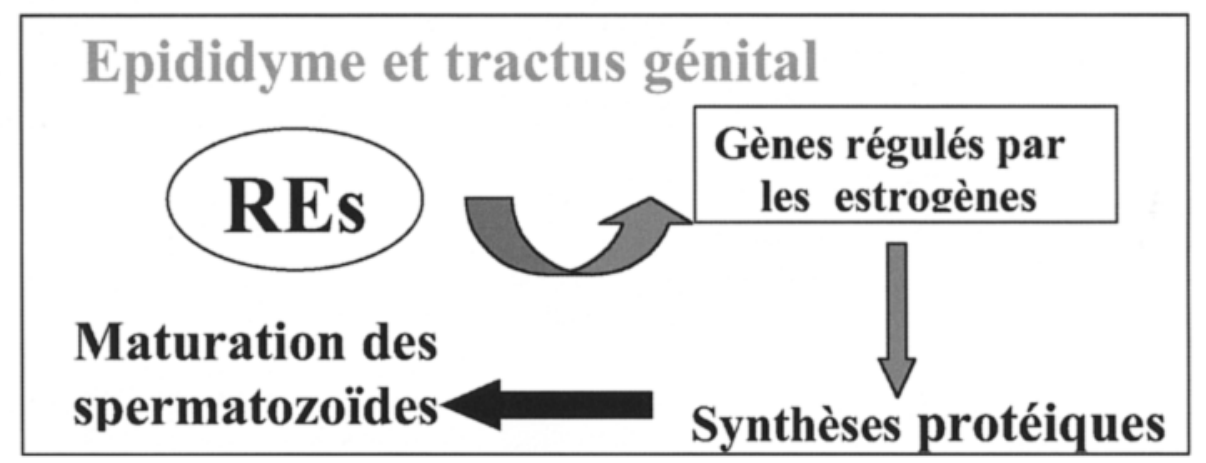

Figure 3 : Représentation schématique : aromatase, estrogènes et maturation du gamète mâle. 
Même s'il reste encore beaucoup à faire concernant le rôle de l'aromatase dans le spermatozoïde, sa présence est clairement établie sur deux sites : région équatoriale de l'acrosome et pièce intermédiaire plus flagelle à un degré moindre. Donc la participation des estrogènes dans la réaction acrosomique, la mobilité et la survie [30] des spermatozoïdes est fortement envisagée. La présence simultanée de l'aromatase et des récepteurs aux estrogènes permet d'émettre l'hypothèse d'une autocrinie des estrogènes endogènes. La localisation de l'aromatase au niveau de la région équatoriale reste à confirmer. Cependant il est intéressant de noter que des auteurs avaient retrouvé le récepteur aux estrogènes dans la même région [36]. Si ces résultats se confirment, des études complémentaires permettraient de savoir si la synthèse d'estrogènes à ce niveau pourrait avoir un rôle dans la réaction acrosomique comme cela avait été suggéré antérieurement $[3,17]$.

\section{Remerciements :}

Les Drs S. Lambard et A. Chocat sont chaleureusement remerciés pour leur aide dans ces travaux qui ont été financés par le Ministère de l'Education Nationale et de la Recherche, et la Région Basse-Normandie.

\section{REFERENCES}

1. AKINGBEMI B.T. : Estrogen regulation of testicular function. Reprod. Biol. Endocrinol., 2005, $3: 51$.

2. AQUILA S., SISCI D., GENTILE M., MIDDEA E., SICILIANO L., ANDO S. : Human ejaculated spermatozoa contain active P450 aromatase. J. Clin. Endocrinol. Metab., 2002, 87 : 3385-3390.

3. AQUILA S., SISCI D., GENTILE M. et al. : Towards a physiological role for cytochrome $\mathrm{P} 450$ aromatase in ejaculated human sperm. Hum. Reprod., 2003, 18 : 1650-1659.

4. AQUILA S., SISCI D., GENTILE M. et al. : Estrogen receptor (ER)a and $\mathrm{ERb}$ are both expressed in human ejaculated spermatozoa : evidence for their direct interaction with phosphatidylinositol-3-OH Kinase/Akt pathway. J. Clin. Endocrinol. Metab., 2004, 89 :14431451.

5. AQUILAS., MIDDEAE., CATALANO S. et al. : Human sperm express a functional androgen receptor : effects on PI3KJAKT pathway. Hum. Reprod., 2007, 22 : 2594-2605.

6. BECK K.J., HERSCEL S., HUNGERSHOFER R., SCHWINGER E.: The effect of steroid hormones on motility and selective migration of $X$-and Y-bearing human spermatozoa. Fertil. Steril., 1976, 27 : 407412.

7. BRODIE A., INKSTER S., YUE W. : Aromatase expression in human male. Mol. Cell. Endocrinol., 2001, 178 : 23-28.

8. CARPINO F., ROMEO F., RAGO F.V. : Aromatase immunolocalization in human ductuli efferentes and proximal ductus epididymis. J. Anat., 2004, $204: 217-220$.

9. CARREAU S. : Paracrine control of human Leydig cell and Sertoli cell functions. Folia Histochem. Cytobiol., 1996, $3: 111-119$.

10. CARREAU S., GENISSEL C., BILINSKA B., LEVALLET J. : The oestrogen sources in the testis and the reproductive tract of the male. Int. J. Androl., 1999, 22 : 211- 223.

11. CARREAU S., LAMBARD S., DELALANDE C., GALERAUD-DENIS I., BILINSKA B., BOURGUIBA S. : Aromatase expression and role of estrogens in male gonad : a review. Reprod. Biol. Endocrinol., 2003, $1: 35$.

12. CARREAU S., DELALANDE C., SILANDRE D., BOURGUIBA S.,
LAMBARD S. : Aromatase and estrogen receptors in male reproduction. Mol. Cell. Endocrinol., 2006, 246 : 65-68.

13. CARREAU S., BOURGUIBA S., DELALANDE C. et al. : Estrogens and male reproduction. Immun. Endocr. Metab. Agents Med. Chem., 2008, $8: 59-65$

14. CARREAU S., de VIENNE C., GALERAUD-DENIS I. : Aromatase and estrogens in man reproduction : a review and latest advances. Adv. Med. Sci., 2008, 53 : sous presse.

15. CHEN J.Q., YAGER J.D., RUSSO J. : Regulation of mitochondrial respiratory chain structure and function by estrogens/estrogen receptors and potential physiological/pathophysiological implications. Biochem. Biophys. Acta, 2005, 1746 : 1-17.

16. DURKEE T.J., MUELLER M., ZINAMAN M. : Identification of estrogen receptor protein and messenger ribonucleic acid in human spermatozoa. Am. J. Obst. Gyn., 1998, 178 :1288-1295.

17. FRASER L.R., BEYRET E., MILLIGAN S.R., ADEOYA-OSIGUWA S.A. Effects of estrogenic xenobiotics on human and mouse spermatozoa. Hum. Reprod., 2006, 21 : 1184-1193.

18. GUNASEGARAM R., CHEW P.C.T., LOGANATH A., PEH K.L., RATMAN S.S. : A delta 4-3 keto pathway for testosterone synthesis in the human spermatozoa. Arch. Androl., 1995, $40: 49-57$.

19. HENDRY W.F., PARSLOW J.M., STEDRONSKA J. : Exploratory scrotomy in 168 azoospermic males. Brit. J. Urol., 1983, 55 : 785791.

20. IDAOMAR M., GUERIN J.F., LORNAGE J., CZYBA J.C. : Stimulation of motility and energy metabolism of spermatozoa from asthenospermic patients by $17 \mathrm{~b}$-estradiol. Arch. Androl., 1989, 22 : 197-202.

21. LAMBARD S., GALERAUD-DENIS I., BOURAIMA H., BOURGUIBA S., CHOCATA., CARREAU S. : Expression of aromatase in human ejaculated spermatozoa : a putative marker of motility. Mol. Hum. Reprod., 2003, $9:$ 117-124.

22. LAMBARD S., GALERAUD-DENIS I., SAUNDERS P.T.K., CARREAU $S$. : Human immature germ cells and ejaculated spermatozoa contain aromatase and oestrogen receptors. J. Mol. Endocrinol., 2004, 32 : 279-289.

23. LAMBARD S., CARREAU S. : Aromatase and estrogens in human male germ cells. Int. J. Androl., 2005, 28 : 254-259.

24. LAMBARD S., SILANDRE D., DELALANDE C., GALERAUD-DENIS I., BOURGUIBAS., CARREAU S. : Aromatase in testis : expression and role in male reproduction. J. Steroid. Biochem. Mol. Biol., 2005, $95: 63-69$

25. LEVALLET J., BILINSKA B., MITTRE H., GENISSEL C., FRESNEL J., CARREAU S. : Expression and immunolocalization of functional cytochrome $\mathrm{P} 450$ aromatase in mature rat testicular cells. Biol. Reprod., 1998, 58 : 919-926.

26. LUCONI M., FRANCAVILLAF., PORAZZI I., MACEROLA B., FORTI G., BALDI I. : Human spermatozoa as a model for studying membrane receptors mediating rapid nongenomic effects of progesterone and estrogens. Steroids, 2004, $69: 553-559$.

27. O'DONNELL L., ROBERTSON K.M., JONES M.E., SIMPSON E.R.: Estrogen and spermatogenesis. Endocr. Rev., 2001, $22: 289-318$.

28. OSTERMEIER G.C., GOODRICH R.J., DIAMOND M.P., DIX D.J., KRAWETZ S.A. : Toxard using stable spermatozoal RNAs for prognostic assesment of male factor fertility. Fertil. Steril., 2005, 83: 1687-1694.

29. PAYNE A.H., KELCH R.P., MUSICH S.S., HALPERN M.E. : Intratesticular site of aromatization in the human. J. Clin. Endocrinol. Metab., 1976, 42 : 1081-1087.

30. PENTIKAINEN V., ERKKILAK., SUOMALAINEN L. et al. : Estradio acts as a germ cell survival factor in the human testsi in vitro. J. Clin. Endocrinol. Metab., 2000, 85 : 2057-2067.

31. PEREYRA-MARTINEZ A.C., ROSELLI C.E., STADELMAN H.L., RESKO J.A : Cytochrome P450 aromatase in testis and epididymis of male rhesus monkeys. Endocrine, 2001,16 : 15-19. 
32. ROCHIRAV., GRANATAA.R.M., MADEO B., ZIRILLI L., ROSSI G., CARANI C. Estrogens in males : what we have learned in last 10 years ? Asian J. Androl., 2005, $7:$ 3-20.

33. RAGO V., BILINSKAB., PALMAA., ANDO S., CARPINOA. : Evidence of aromatase localization in cytoplasmic droplet of human immature ejaculated spermatozoa. Folia Histochem. Cytobiol., 2003, 41 : $23-$ 28.

34. SEBASTIAN S., BULUN S.E. : A highly complex organization of the regulatory region of the human CYP19 (aromatase) gene revealed by the Human genome project. J. Clin. Endocrinol. Metab., 2001, 86: 4600-4602.

35. SIMPSON E.R., MAHENDROO M.S., MEANS G.D. et al. : Aromatase cytochrome $\mathrm{P} 450$, the enzyme responsible for estrogen biosynthesis. Endocr. Rev., 1994, 15 : 342-355.

36. SOLAKIDI S., PSARRAA.M.G., NIKOLAROPOULOS S., SEKERIS C.E. : Estrogen receptors alpha and beta (ERalpha and ERbeta) and androgen receptor (AR) in human sperm : localization of ERbeta and $A R$ in mitochondria of the midpiece. Hum. Reprod., 2005, 20 : 3481-3485.

37. TOPPARI J., LARSEN J.C., CHRISTIANSEN P. et al. : Male reproductive health and environmental xenoestrogens. Environm. Health Perspect., 1996, $104: 741-803$.

38. TURNER K.J., MacPHERSON S., MILLAR M.R. et al. : Development and validation of a new monoclonal antibody to mammalian aromatase. J. Endocrinol., 2002, $172: 21-30$.

Manuscrit reçu : septembre 2008 ; accepté septembre 2008.

Communication présentée au XXVème congrès de la SALF, Hammamet 2008

ABSTRACT

Aromatase and male infertility

Serge CARREAU, Claire de VIENNE, Lamia SAID, Ali SAAD, Isabelle GALERAUD-DENIS

The mammalian testis is a complex organ which produces spermatozoa and synthesizes steroids. The transformation of androgens into estrogens is catalyzed by aromatase, an enzymatic complex encoded by a single copy-gene (cyp19) which contains 18 exons, 9 of which are translated. In man, in addition to Leydig cells, we have demonstrated the existence of a biologically active aromatase in immature germ cells and in ejaculated spermatozoa. The presence of estrogen receptors (ERa and ERß) in immature germ cells and spermatozoa has also been reported.

A $30 \%$ decrease in the amount of aromatase mRNA is observed in immotile sperm compared to the motile sperm fraction from the same sample.

Aromatase and GAPDH transcript levels have also been determined by real-time PCR following RNA extraction in sperm samples from individual ejaculates of normozoospermic, and infertile patients. The aromatase protein and markers of either acrosome (CD 46), or tail (tubulin) or mid-piece (Mitotracker Green) or nucleus (DAPI) were immunolocalized by confocal microscopy.

In asthenoteratozoospermic, teratozoospermic and asthenozoospermic patients, aromatase gene expression is decreased by $67 \%, 52 \%$ and $44 \%$, respectively, compared to normospermic controls. Statistical analysis between sperm morphology and aromatase/GAPDH ratio revealed a high degree of correlation ( $r=-0.64$ ) between this ratio and the percentage of abnormal spermatozoa (especially microcephaly).

Using confocal microscopy, we have observed for the first time the existence of two sites for aromatase: the first site co-localized with tubulin is the tail with intensive staining in the mid-piece and the second site corresponds to the equatorial region of the head. Decreased sperm count and motility have been reported in genetically aromatase-deficient men.

In addition to gonadotrophins and testosterone, estrogens therefore also probably play a relevant role in spermatogenesis and human male gamete maturation. Our data suggest that aromatase transcripts could be a marker of male gamete quality and the existence of two sites of aromatase could be related to the role of estrogens in motility and acrosome reaction. Male infertility is a worldwide social problem and consequently, comparative studies of mRNA fingerprints in ejaculates between fertile and infertile men may contribute to elucidate some aspects of male reproductive pathology.

Key words : aromatase, estrogens, spermatozoa, testis, man, fertility 